\title{
A Spectrally Efficient Modified Asymmetrically and Symmetrically Clipped Optical (mASCO)-OFDM for IM/DD Systems
}

Mohammed Salman Baig ( $\sim$ salmanbaig.mmantc@gmail.com )

Maulana Mukhtar Ahmad Nadvi Technical Campus https://orcid.org/0000-0002-7624-3899

Mohammed Thamer ALRESHEEDI

King Saud University

Mohd Adzir MAHDI

UPM: Universiti Putra Malaysia

Ahmad Fauzi ABAS

King Saud University

Research Article

Keywords: ACO-OFDM, SCO-OFDM, ASCO-OFDM, mSCO-OFDM

Posted Date: February 25th, 2022

DOI: https://doi.org/10.21203/rs.3.rs-1342257/v1

License: (9) This work is licensed under a Creative Commons Attribution 4.0 International License.

Read Full License 


\title{
A spectrally efficient modified asymmetrically and symmetrically clipped optical (mASCO)-OFDM for IM/DD systems
}

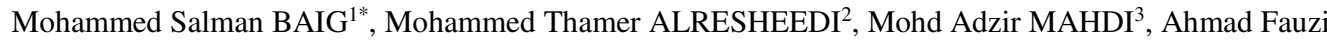 \\ $\mathrm{ABAS}^{2}$ \\ ${ }^{1}$ Department of Computer Engineering, Maulana Mukhtar Ahmed Nadvi Technical Campus, Malegaon \\ 423203, Maharashtra, India \\ ${ }^{2}$ Department of Electrical Engineering, College of Engineering, P.O. Box 800, King Saud University, \\ Riyadh 11421, Kingdom of Saudi Arabia \\ ${ }^{3}$ Department of Computer and Communication Systems, Faculty of Engineering, Universiti Putra \\ Malaysia, 43400 UPM Serdang, Selangor, Malaysia \\ *Corresponding author: salmanbaig.mmantc@gmail.com
}

\begin{abstract}
A novel spectrally efficient modified asymmetrically and symmetrically clipped optical (mASCO)OFDM for intensity modulated direct detection (IM/DD) systems is presented. The conventional ASCO-OFDM systems uses two frame to transmit the conventional asymmetrically clipped optical (ACO)-OFDM and symmetrically clipped optical (SCO)-OFDM system. The proposed mASCOOFDM system replaces the two frame SCO-OFDM by a single frame modified SCO (mSCO)-OFDM. The mSCO-OFDM clips the data only one side of the symmetry and performs an absolute function on the other side of the symmetry. This allows mASCO-OFDM to be 1.333 times more spectral efficient than the conventional ASCO-OFDM. The mASCO-OFDM reduces its receiver's complexity by estimating and eliminating the clipping noise distortion in time domain. Overall, this system has $43 \%$ lower complexity in comparison to ASCO-OFDM system. The mASCO-OFDM shows a better BER performance and a lower Peak Average Power Ratio (PAPR) than ASCO-OFDM. The proposed system also shows better BER performance than ACO-OFDM for the same spectral efficiency.
\end{abstract}

Keywords: ACO-OFDM, SCO-OFDM, ASCO-OFDM, mSCO-OFDM

\section{Introduction}

Orthogonal frequency division multiplexing (OFDM) has been successfully ap-plied to intensity modulated/direct detection (IM/DD) systems. DC-bias optical OFDM (DCOOFDM) [1-3] and asymmetrically clipped optical OFDM (ACO-OFDM) [1, 2, 4] are basic forms of optical OFDM (O-OFDM) techniques that generate real and unipolar 
signals required for (IM/DD) transmission mediums. DCO-OFDM has 100\% spectral efficiency (SE) as it uses all its subcarriers to process its information. However, the DC bias in DCO-OFDM is inefficient in terms of optical power [5]. As ACO-OFDM is more power efficient it is generally preferred over DCO-OFDM for IM/DD applications. The SE of ACO-OFDM is $50 \%$ as it processes its data only on its odd subcarriers.

This motivated the researchers to enhance the SE of ACO-OFDM. A hybrid scheme named ADO-OFDM was developed in [1], that utilized all subcarriers by combining ACO-OFDM on its odd subcarriers and DCO-OFDM on even sub-carriers. Another hybrid technique known as Hybrid ACO-OFDM (HACO-OFDM) [6] combined ACO-OFDM on odd subcarriers and PAM-DMT on the imaginary parts of the even ones. This scheme is spectrally inefficient, as the real parts of even subcarriers remain unused. To overcome this disadvantage, Enhanced hybrid asymmetrically clipped optical OFDM (EHACO-OFDM) [7] added a third path of DCO-OFDM on the unused real parts of even subcarriers of HACO-OFDM. EHACO-OFDM achieved higher power efficiency than ADO-OFDM and DCO-OFDM, however it was still optically inefficient due to DCO-OFDM.

The power inefficient DCO-OFDM was replaced by a technique known as symmetrically clipped optical OFDM (SCO-OFDM) that used two frame flip type processing to carry the negative and positive information in ASCO-OFDM [5]. Clipping directly on even subcarriers resulting in loss of information due to even symmetry, is prevented by the two frame SCO-OFDM in ASCO-OFDM. This processing also requires a two frame ACO-OFDM to process its information, thereby increasing its bandwidth and complex processing at the receiver. ASCO-OFDM achieved better symbol error rate (SER) performance and lower optical power per bit in comparison to ADO-OFDM [5] at the expense of a large bandwidth with increased complexity at the receiver. The SE of ASCO-OFDM is $75 \%$ of SE of DCO-OFDM.

It has been proven that the clipping distortion of ACO-OFDM falls on the even subcarriers without effecting its data on odd subcarriers. The conventional receivers in $[1,5,8,9]$ use complex signal processing by combining both frequency-domain and time-domain processing to estimate and cancel the clipping distortion of ACO-OFDM. This process becomes more significant when subsequent streams of even subcarriers carry additional information, as its BER performance depends on the accurate estimation and cancellation of clipping distortion of ACO-OFDM. Many iterative procedures have also been used to cancel the clipping distortion, that have shown to improve the BER performance but at the cost of increased complexity [8].

In this paper, modified ASCO-OFDM (mASCO-OFDM) system is proposed for IM/DD optical wireless systems. The proposed mASCO-OFDM utilizes all the subcarriers and has the same SE of DCO-OFDM. The simplified model mASCOOFDM replaces the two frame SCO-OFDM by a proposed single frame modified SCO (mSCO)-OFDM signal, that clips only one side of the symmetry and performs an absolute function of the data on the other side of the symmetry. The clipped data contains both the data and the clipping distortion (represented by the absolute function of the data) on the even subcarriers. The absolute function on the other side of the 
symmetry will help eliminate the clipping distortion and accurately recover the data at the receiver. This system additionally applies the concept of time domain clipping noise estimation (TD-CNE) [10] to its receiver to recover its signal information through timedomain signal processing that reduces the receiver complexity and further improves the overall BER performance of mASCO-OFDM signal.

\section{System Modeling}

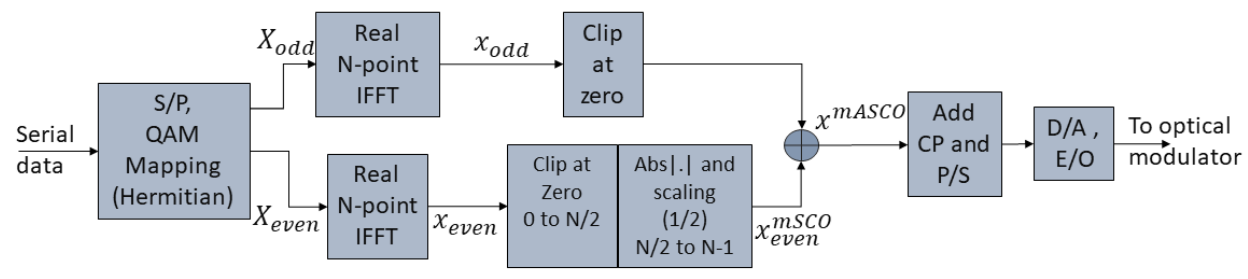

Fig. 1 mASCO-OFDM Transmitter

Fig. 1 shows the transmitter of mASCO-OFDM system. The serial to parallel (S/P) data are mapped using QAM symbols and then constrained to Hermitian symmetry. The resulting signal $X_{K}$ is divided into odd and even stream of subcarriers, where

$$
X_{\text {odd }}=\left[\begin{array}{c}
0, X_{o d d, 1}, 0, X_{o d d, 3}, 0, \ldots, X_{o d d, \frac{N}{2}-1}, 0 \\
X_{o d d, \frac{N}{2}-1}{ }^{*}, \ldots, X_{o d d, 3}{ }^{*}, 0, X_{o d d, 1}{ }^{*}, 0
\end{array}\right]
$$

where $X_{o d d, K}=X_{K}$ is a complex QAM symbol for $K=1,3 \ldots, \frac{N}{2}-1$ and $N$ is the number of subcarriers. The Hermitian constrained $X_{\text {odd }}$ input block to the IFFT block generates real time domain signal $x_{o d d, n}$ has an anti-symmetric property [2, 4].This signal is zero clipped to yield the unipolar conventional ACO-OFDM signal generated from odd subcarriers denoted as $x_{\text {odd }}^{A C O}$ generated as

$$
x_{o d d}^{A C O}=\left\{\begin{array}{cl}
\left\lfloor x_{o d d, n}\right\rfloor_{C} & 0 \leq n \leq \frac{N}{2}-1 \\
\left\lfloor-x_{o d d, n-N / 2}\right\rfloor_{C} & N / 2 \leq n \leq N-1
\end{array}\right.
$$

where $\left\lfloor x_{o d d, n}\right\rfloor_{C}=\max \left\{x_{o d d, n}, 0\right\}\left([.\rfloor_{c}\right.$ denotes the zero bias clipping operation) [6, $11]$ and can also be represented as

$$
\left\lfloor x_{o d d, n}\right\rfloor_{c}=\frac{1}{2}\left(x_{o d d, n}+\left|x_{o d d, n}\right|\right)
$$


where the clipping distortion $\left|x_{o d d, n}\right|$ falls on the even subcarriers and does not affect the data $x_{o d d, n}$ on the odd subcarriers $[1,4]$.

The even stream of subcarriers $X_{\text {even }}$ are represented as

$$
X_{\text {even }}=\left[\begin{array}{l}
0,0, X_{\text {even }, 1,}, 0, X_{\text {even }, 2}, \ldots, X_{\text {even }, \frac{N}{4}} \\
0,0,0, X_{\text {even }, N / 4}{ }^{*}, \ldots, 0, X_{\text {even }, 0}{ }^{*}, 0
\end{array}\right]
$$

where $X_{\text {even }, K}=\left(X_{K}\right)$ is a complex QAM symbol for $K=2,4 \ldots, \frac{N}{2}-1$ The first and $\left(\frac{N}{2}\right)$ th subcarriers are not modulated to fulfill the Hermitian symmetry requirements. This resultant signal when input to the IFFT block generates real time domain signal $x_{\text {even }}$ and has a symmetric property. Since, it has a symmetric property, direct negative clipping of this signal causes loss of information [5]. Alternatively, across the symmetry, clipping is done only one side of the symmetry. The absolute value of the data is performed at the other side of the symmetry and is scaled by half. The resulting signal $x_{\text {even }}^{m S C O}$ is given as,

$$
x_{\text {even }}^{m S C O}=\left\{\begin{array}{cc}
\left\lfloor x_{\text {even }, n}\right\rfloor_{C} & 0 \leq n \leq \frac{N}{2}-1 \\
\frac{1}{2}\left|x_{\text {even }, n-N / 2}\right| & \frac{N}{2} \leq n \leq N-1
\end{array}\right.
$$

where $\left|x_{\text {even, } n-N / 2}\right|$ represents the absolute value function (|.|) and $\left\lfloor x_{\text {even }, n}\right\rfloor_{C}=\max \left\{x_{\text {even }, n}, 0\right\}$ (zero bias clipping operation) is also expressed as

$$
\left\lfloor x_{\text {even }, n}\right\rfloor_{C}=\frac{1}{2}\left(x_{\text {even }, n}+\left|x_{\text {even }, n}\right|\right)
$$

where the data $x_{\text {even,n }}$ and the clipping distortion $\left(\left|x_{\text {even, } n}\right|\right)$ are present on the even subcarriers from $0 \leq n \leq \frac{N}{2}-1$.

The generated real time domain signals $x_{\text {odd }}^{A C O}$ and $x_{\text {even }}^{m S C O}$ are combined to yield a real valued non-negative signal $x_{m A S C O, m}$ given as

$$
x^{m A S C O, n}=x_{o d d}^{A C O}+x_{e v e n}^{m S C O}
$$

The combined signal $x^{m A S C O, n}$ consists of the undistorted ACO-OFDM signal on the odd subcarriers. The even subcarriers on one side of the symmetry contains the mSCOOFDM data and the clipping distortions of ACO-OFDM and mSCO-OFDM, while the other side contains only the absolute value of mSCO-OFDM data and clipping distortion of ACO-OFDM.

The combined signal $x^{m A S C O, n}$ in Equation (7) is appended with a Cyclic Prefix (CP) and converted from parallel to serial (P/S). This is followed by the optical modulation 
process across a flat channel as in $[1,5,12]$. The shot noise and thermal noise are modeled as additive white Gaussian noise (AWGN) as in $[1,5,9,12,13]$.

At the Receiver (Fig. 2), the received signal is first converted from an optical to electrical signal using a photodiode and then converted from analog to digital (A/D). The resulting received signal after removing the $\mathrm{CP}$ and parallel to serial conversion $(\mathrm{P} / \mathrm{S})$ is given as

$$
y^{m A S C O, n}=x^{m A S C O, n}+\mathrm{w}_{n}
$$

where $\mathrm{w}_{n}$ is modeled as the AWGN.

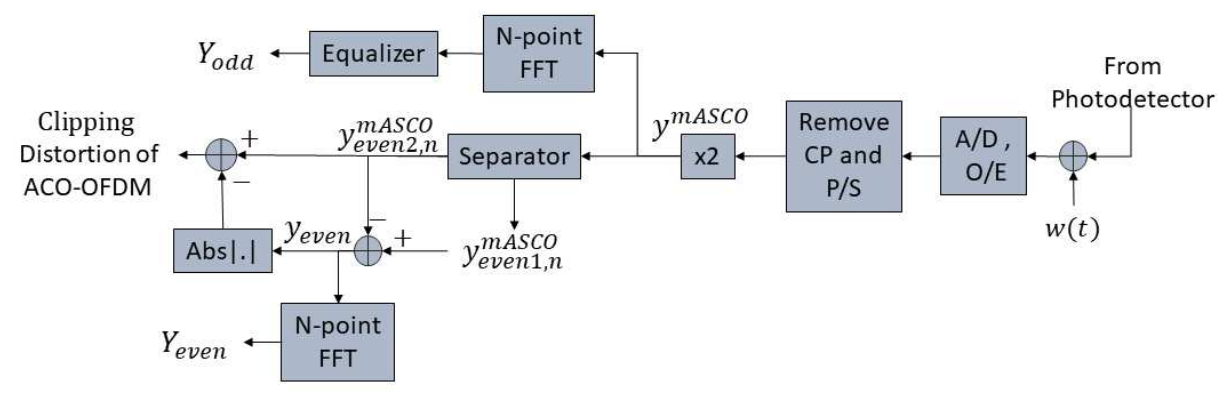

Fig.2 mASCO-OFDM Receiver

The mASCO-OFDM receiver implements TD-CNE [10] so that the data retrieval from the even subcarriers can be done independently.

$$
\begin{aligned}
& y^{m A S C O, n}= \\
& \begin{cases}\left\lfloor x_{\text {odd }, n}\right\rfloor_{C}+\left\lfloor x_{\text {even }, n}\right\rfloor_{C}+\mathrm{w}_{\text {odd }, n}+\mathrm{w}_{\text {even }, n} & 0 \leq n \leq \frac{N}{2}-1 \\
\left.\mid-x_{\text {odd }, n-\frac{N}{2}}\right\rfloor_{C}+\left|x_{\text {even }, n-\frac{N}{2}}\right|-\mathrm{w}_{\text {odd }, n-\frac{N}{2}}+\mathrm{w}_{\text {even }, n-\frac{N}{2}} & \frac{N}{2} \leq n \leq N-1\end{cases}
\end{aligned}
$$

where $\mathrm{w}_{\text {odd,n }}$ and $\mathrm{w}_{\text {even,n }}$ are the AWGN that contribute to the odd and even subcarriers respectively. Inserting Eq. (3) and (6) into Eq. (9), and scaling by a factor of 2 , yields

$$
\begin{aligned}
& y^{m A S C O, n}= \\
& \left\{\begin{array}{lc}
\left(x_{\text {odd }, n}+\left|x_{\text {odd }, n}\right|+x_{\text {even }, n}+\left|x_{\text {even }, n}\right|\right)+\mathrm{w}_{\text {odd } 1, n}+\mathrm{w}_{\text {even } 1, n} & 0 \leq n \leq \frac{N}{2}-1 \\
\left(-x_{\text {odd }, n-\frac{N}{2}}+\left|-x_{\text {odd }, n-\frac{N}{2}}\right|+\left|x_{\text {even }, n}\right|\right)+\mathrm{w}_{\text {odd } 2, n}+\mathrm{w}_{\text {even }, n} & \frac{N}{2} \leq n \leq N-1
\end{array}\right.
\end{aligned}
$$


As no clipping distortion falls on odd subcarriers, it can be directly estimated from $y_{\text {odd, } n}^{\text {mASCO as }}$

$$
y_{o d d, n}=y_{o d d, n}^{m A S C O}=x_{o d d, n}+\mathrm{w}_{o d d, n}
$$

This is then input to the FFT and equalized to recover the odd subcarriers symbols $Y_{\text {odd }}$ of QAM constellation.

The even subcarriers of scaled version of received signal $\mathrm{y}_{m A S C O, n}$ is split into $y_{\text {even } 1, n}^{m A S C O}$ and $y_{\text {even } 2, n}^{\text {mASCO }}$ where

$$
\begin{aligned}
& y_{\text {even } 1, n}^{\text {mASCO }}=y_{\text {even }, n}^{\text {mASCO }}=\left|x_{\text {odd }, n}\right|+x_{\text {even }, n}+\left|x_{\text {even }, n}\right|+\mathrm{w}_{\text {even } 1, n} \\
& y_{\text {even } 2, n}^{\text {mASCO }}=y_{\text {even }, n+N / 2}^{m A S C O}=\left|x_{\text {odd }, n}\right|+\left|x_{\text {even }, n}\right|+\mathrm{w}_{\text {even } 2, n}
\end{aligned}
$$

Subtracting Equations (12) and (13), yields the even signal in time domain as

$$
\begin{aligned}
& y_{\text {even }, n}=y_{\text {even } 1, n}^{\text {mASCO }}-y_{\text {even } 2, n}^{m A S C O} \\
& =\left|x_{\text {odd }, n}\right|+x_{\text {even }, n}+\left|x_{\text {even }, n}\right|+\mathrm{w}_{\text {even } 1, n} \\
& \quad-\left|x_{\text {odd }, n}\right|-\left|x_{\text {even }, n}\right|-\mathrm{w}_{\text {even } 2, n} \\
& =x_{\text {even }, n}
\end{aligned}
$$

As a flat channel is considered, the noise is $\mathrm{w}_{\text {even } 1, n}=\mathrm{w}_{\text {even } 2, n}$ due to symmetry, and will thus cancel each other. This signal is then transformed into frequency domain, from which the even symbols of QAM constellation are recovered.

\section{Performance Evaluation}

\subsection{Complexity Analysis}

The complexity of the system is determined by the number of complex multiplications of FFT/IFFT operations [14].

The computational complexity for ASCO-OFDM transmitter is $3 \mathcal{O}\left(\mathrm{Nlog}_{2} \mathrm{~N}\right)$ as it requires two IFFT's for the stream of odd subcarrier, and one IFFT for even subcarriers. The computational complexity of ASCO-OFDM receiver is $4 \mathcal{O}\left(\mathrm{Nlog}_{2} \mathrm{~N}\right)$ as it requires two FFT's and IFFT's for the demodulation of its signals. 
The computational complexity for the proposed model mASCO-OFDM transmitter is $2 \mathcal{O}\left(\mathrm{Nlog}_{2} \mathrm{~N}\right)$, as it requires two $\mathrm{N}$-point IFFT for its odd and even stream of subcarriers. The computational complexity for the proposed receiver of mASCOOFDM is $2 \mathcal{O}\left(\mathrm{Nlog}_{2} N\right)$.

The complexity analysis for mASCO-OFDM and ASCO-OFDM is summarized in Table 1

Table 1. Computational Complexity

\begin{tabular}{ccc}
\hline OFDM schemes & Transmitter & Receiver \\
\hline \hline ASCO-OFDM & $3 \mathcal{O}\left(N \log _{2} N\right)$ & $4 \mathcal{O}\left(N \log _{2} N\right)$ \\
mASCO-OFDM & $2 \mathcal{O}\left(N \log _{2} N\right)$ & $2 \mathcal{O}\left(N \log _{2} N\right)$ \\
\hline
\end{tabular}

The complexity reduction ratio is evaluated to determine the improvement for the proposed system in comparison to the conventional system. The $\mathrm{Cr}$ is evaluated as

$$
C r=\left(1-\frac{\text { Cproposed }}{\text { Cconventional }}\right) * 100 \%
$$

mASCO achieves 33\% lower complexity at the transmitter and 50\% lower complexity at the receiver. Thus the overall complexity of mASCO-OFDM reduces to about $43 \%$ in comparison to the conventional ASCO-OFDM.

\subsection{Spectral efficiency}

The SE for ACO-OFDM for a large 'N' IFFT size without the CP can be approximated as

$$
\gamma_{A C O-O F D M} \approx \frac{\left(\frac{N}{2}\right) \log _{2} Q_{A C O}}{N} \approx \frac{\log _{2} Q_{A C O}}{2} \quad \text { bits } / \mathrm{sec} / \mathrm{Hz}
$$

where $Q_{A C O}$ represent the constellation sizes.

The SE approximation for ASCO-OFDM for a large ' $N$ ' IFFT size is given by

$$
\begin{aligned}
\gamma_{A S C O-O F D M} & \approx \frac{\left(\frac{N}{2}\right) \log _{2} Q_{A C O}+\left(\frac{N}{2}-1\right) \frac{1}{2} \log _{2} Q_{S C O}}{N} \\
& \approx \frac{\log _{2} Q_{A C O}+\frac{1}{2} \log _{2} Q_{S C O}}{2} \text { bits } / \mathrm{sec} / \mathrm{Hz}
\end{aligned}
$$

where $Q_{A C O}$ and $Q_{S C O}$ represent the constellation sizes of ACO-OFDM and SCOOFDM respectively.

For a large 'N' IFFT size, the SE approximation for the proposed model is given as 


$$
\begin{aligned}
\gamma_{m A S C O-O F D M} & \approx \frac{\left(\frac{N}{2}\right) \log _{2} Q_{A C O}+\left(\frac{N}{2}-1\right) \log _{2} Q_{m S C O}}{N} \\
& \approx \frac{\log _{2} Q_{A C O}+\log _{2} Q_{m S C O}}{2} \quad \text { bits } / \mathrm{sec} / \mathrm{Hz}
\end{aligned}
$$

where $Q_{A C O}$ and $Q_{m S C O}$ represent the constellation sizes of the ACO-OFDM and mSCO-OFDM streams respectively.

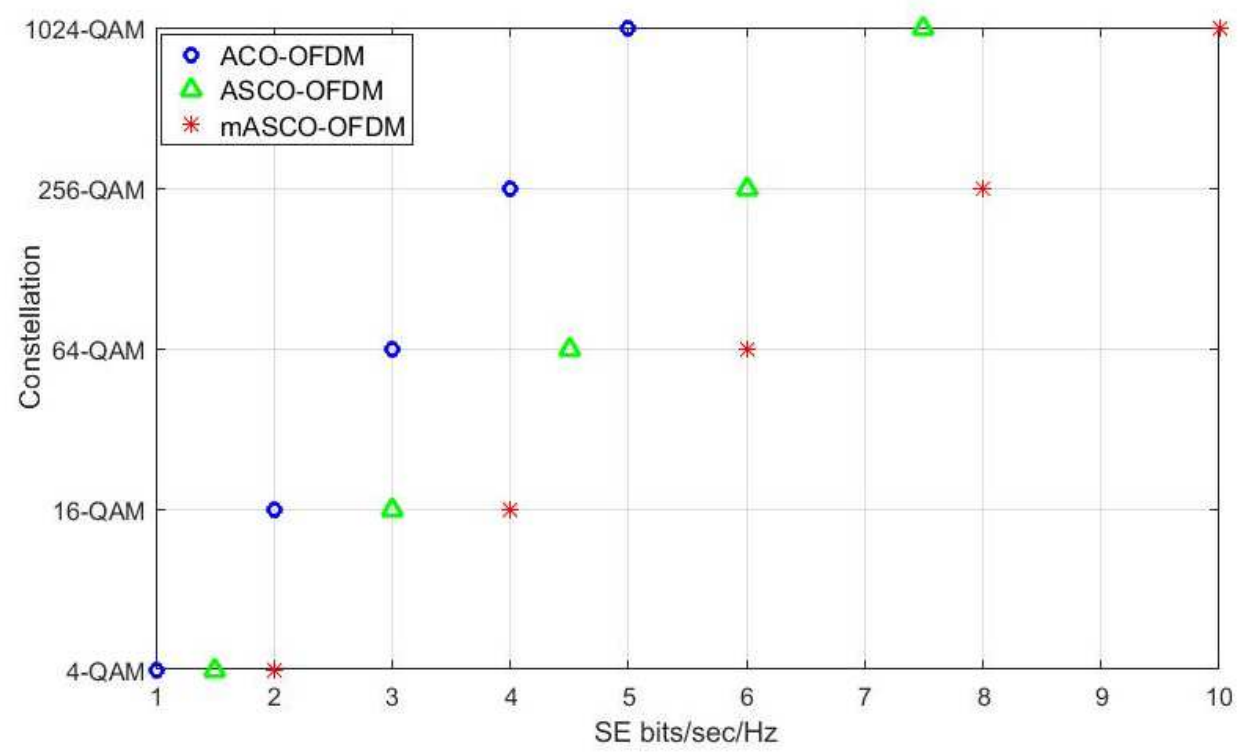

Fig. 3 Constellation vs SE comparison for ACO-OFDM, ASCO-OFDM and mASCO-OFDM

The SE's for a large $\mathrm{N}$ are evaluated and compared against different QAM constellations for ACO-OFDM, ASCO-OFDM and mASCO-OFDM as shown in Fig. 3. For this analysis, same constellation size and equal optical power were used for both odd and even subcarriers for the above mentioned systems. In general, mASCO-OFDM requires a lower QAM modulation to achieve the same spectral efficiency in comparison to ASCO-OFDM and ACO-OFDM. For example, mASCO-OFDM requires 16-QAM (applied to both odd and even streams) and ACO-OFDM requires 256-QAM to achieve same SE of 4bits/sec/Hz. Similarly, ASCO-OFDM requires 256QAM while mASCO-OFDM only requires 64-QAM result to achieve the same SE of 6bits/sec/Hz. These observations can be analyzed by referring to Equations (16), (17) and (18). Theoretically, compared to ACO-OFDM, mASCO-OFDM requires 16 times lower QAM, which means it requires lower bandwidth to achieve the same SE. Comparing with ASCO-OFDM, mASCO-OFDM requires 4 times lower QAM to achieve the same SE. Analyzing for the same QAM, mASCO-OFDM achieves respectively 2 and 1.333 times higher SE than ACO-OFDM and ASCO-OFDM. 


\section{Results}

The simulation results presented in this section were based on the assumption of a perfect synchronization over an AWGN channel.

\subsection{Peak Average Power Ratio (PAPR)}

The non-linear characteristics of optical devices in IM/DD optical system can cause high PAPR that can deteriorate the system. Hence, PAPR is an important parameter in evaluation of an O-OFDM system [12]. Some of the precoding techniques mentioned in [15] have reduced the PAPR, without affecting the bit error rate (BER) performance. The complimentary cumulative distribution function (CCDF) of PAPR for mASCOOFDM and ASCO-OFDM are compared as shown in Fig.4. The total average optical power of each OFDM scheme is normalized to unity as demonstrated in $[1,12]$ for the schemes in Fig. 4. The simulations were performed for 16-QAM on the odd and even subcarriers for conventional ASCO-OFDM and mASCO-OFDM respectively. The conventional ACO-OFDM was also simulated for 16-QAM and is used as a reference baseline model.

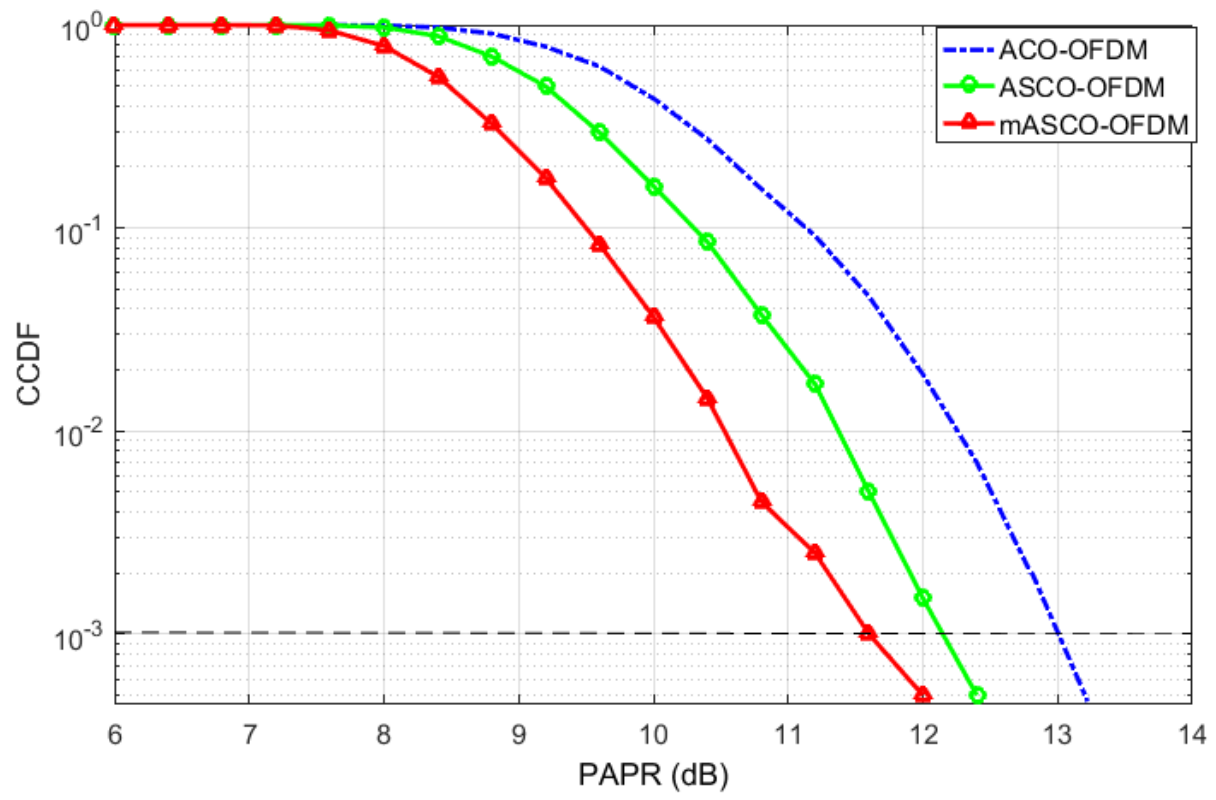

Fig. 4 CCDF of PAPR comparison curves for ACO-OFDM, ASCO-OFDM and mASCO-OFDM

As observed from Fig. 4, the CCDF of the PAPR for mASCO-OFDM is about 0.65 $\mathrm{dB}$ and $1.34 \mathrm{~dB}$ lower than ASCO-OFDM and the conventional ACO-OFDM respectively. This shows that the proposed mASCO-OFDM has a better resilience to the system non-linearity. 


\subsection{Bit-Error-Rate (BER)}

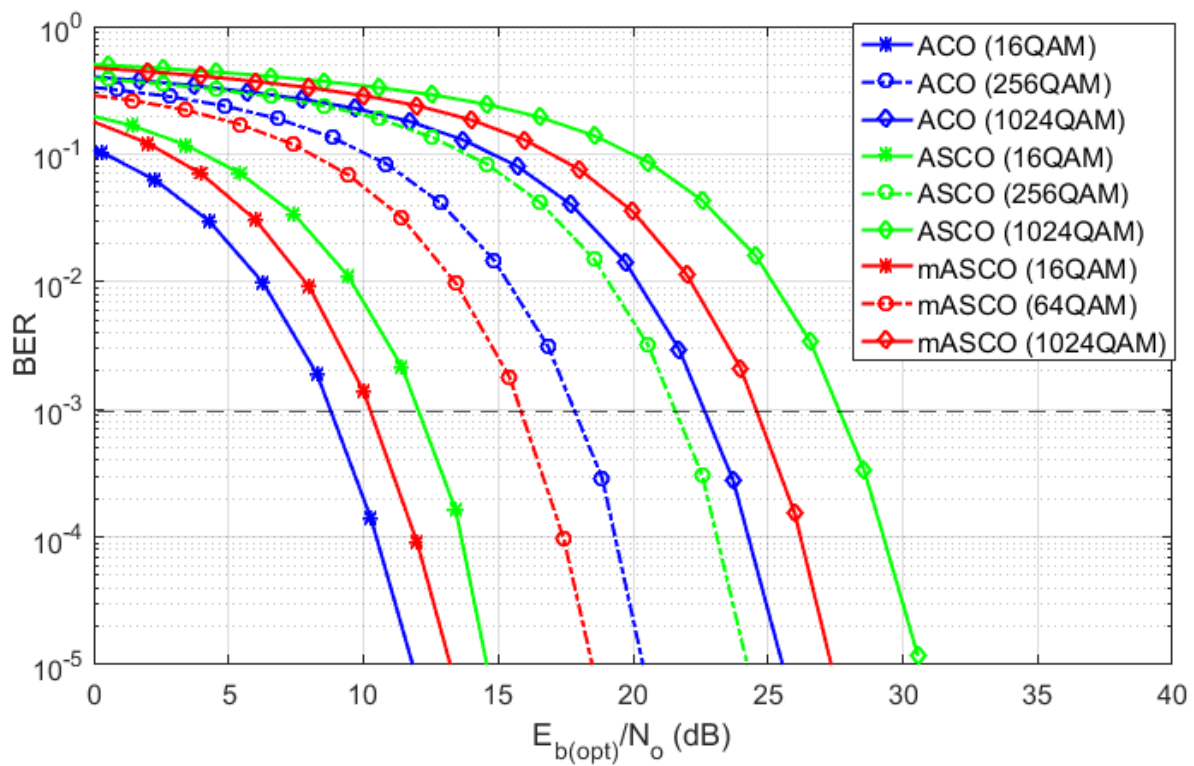

Fig. 5 BER vs optical bit energy to noise ratio performance for ACO-OFDM, ASCO-OFDM and mASCOOFDM

Fig. 5 shows BER against optical bit energy to noise power ratio $\left(E_{b(o p t)} / N_{o}\right)$. The simulations were carried for 16-QAM and 1024-QAM, and same constellations were used for both odd and even streams. The $E_{b(o p t)} / N_{o}$ is expressed as $E_{b(o p t)} / N_{o}=$ $P_{\text {opt }} / b$, where ' $P_{\text {opt }}$ ' is the optical power of the transmitted signal and ' $b$ ' is the bit rate. The optical power $P_{o p t}$ is set to unity for all the models presented in Fig. 5 . The size of the IFFT/FFT is $\mathrm{N}=1024$ and 256 symbols were used.

As evident in Fig. 5, the proposed $\mathrm{mASCO}-\mathrm{OFDM}$ has a superior BER performance than ASCO-OFDM. At the BER of $10^{-3}$, mASCO-OFDM gains $1.73 \mathrm{~dB}$ and $3.12 \mathrm{~dB}$ lower $E_{b(o p t)} / N_{o}$ than conventional ASCO-OFDM for 16-QAM and 1024-QAM respectively. The performance improvement is significantly due to the reduction of errors in mSCO-OFDM, which was evident by the implementation of TD-CNE, as it did not require the estimation and cancellation of the clipping noise of ACO-OFDM.

From this result, it is observed that the proposed mASCO-OFDM showed better BER performance than ASCO-OFDM for the same QAM and SE. However, in comparison to ACO-OFDM this advantage could only be seen for the same SE, and not for the same QAM modulation. For instance, only for the same SE of 4bits/sec/Hz, mASCO-OFDM gains $6 \mathrm{~dB}$ lower $\boldsymbol{E}_{\boldsymbol{b}(\boldsymbol{o p t})} / \boldsymbol{N}_{\boldsymbol{o}}$ than ACO-OFDM. 


\section{Conclusion}

A novel mASCO-OFDM is successfully presented. The proposed system achieves an overall lower computational complexity and higher SE in comparison to conventional ASCO-OFDM. By exhibiting a better BER performance and lower PAPR in comparison to the conventional ASCO-OFDM, the proposed mASCO-OFDM can be a better alternative for IM/DD systems. The performance of mASCO-OFDM were carried out assuming an ideal flat AWGN channel. The proposed model will be further investigated and analyzed in the case of multipath channel in the future work.

Acknowledgements - This work is supported by grant \#RSP-2021/336, King Saud University, Riyadh, Saudi Arabia

\section{References}

[1] Dissanayake, S.D. and J. Armstrong, Comparison of aco-ofdm, dco-ofdm and ado-ofdm in im/dd systems, Journal of lightwave technology, 2013, 31(7), pp. 1063-1072.

[2] Armstrong, J. and B.J. Schmidt, Comparison of asymmetrically clipped optical OFDM and DC-biased optical OFDM in AWGN,Communications Letters, IEEE, 2008, 12(5), pp. 343-345.

[3] Armstrong, J. and A. Lowery, Power efficient optical OFDM, Electron. Lett, 2006, 42(6), pp. 370-372. [4] Armstrong, J., et al. Spc07-4: Performance of asymmetrically clipped optical ofdm in awgn for an intensity modulated direct detection system, in IEEE Global Telecommunications Conference, 2006, GLOBECOM'06.

[5] Wu, N. and Y. Bar-Ness, A novel power-efficient scheme asymmetrically and symmetrically clipping optical (ASCO)-OFDM for IM/DD optical systems, EURASIP Journal on Advances in Signal Processing, 2015, 2015(1), pp. 1-10.

[6] Ranjha, B. and M. Kavehrad, Hybrid Asymmetrically Clipped OFDM-Based IM/DD Optical Wireless System. Journal of Optical Communications and Networking, 2014, 6(4), pp. 387.

[7] Guan, R., et al. Enhanced hybrid asymmetrically clipped orthogonal frequency division multiplexing for optical wireless communications, SPIE 2016, 55(5), 056111 .

[8] Wang, Q., Z. Wang, and L. Dai, Iterative receiver for hybrid asymmetrically clipped optical OFDM. Journal of Lightwave Technology, 2014, 32(22), pp. 3869-3875.

[9] Lowery, A.J., Comparisons of spectrally-enhanced asymmetrically-clipped optical OFDM systems. Optics Express, 2016, 24(4), pp. 3950-3966.

[10] Wang, T., Y. Hou, and M. Ma, A Novel Receiver Design for HACO-OFDM by Time-Domain Clipping Noise Elimination, IEEE Communications Letters, 2018, 22(9), pp. 1862-1865.

[11] Wang, Q., et al., Layered ACO-OFDM for intensity-modulated direct-detection optical wireless transmission. Optics Express, 2015, 23(9), pp. 12382-12393.

[12] Baig, M.S., et al. IM/DD dual stream asymmetrically clipped optical OFDM system, SPIE, 2018, 57(8), 086103.

[13] Yang, Q., A. Al Amin, and W. Shieh, Optical OFDM basics, in Impact of Nonlinearities on Fiber Optic Communications, Springer, 2011, pp. 43-85.

[14] Islim, M.S. and H. Haas, Augmenting the spectral efficiency of enhanced PAM-DMT-based optical wireless communications. Optics express, 2016, 24(11), pp. 11932-11949.

[15] Mohammed, N., M. Elnabawy, and A. Khalaf, PAPR reduction using a combination between precoding and non-liner companding techniques for ACO-OFDM-based VLC systems, Opto-Electronics Review, 2021, pp. 59-70. 